\title{
THE ORIGIN OF [O II] EMISSION IN RECENTLY QUENCHED ACTIVE GALACTIC NUCLEUS HOSTS
}

\author{
Dale D. Kocevski, Brian C. Lemaux ${ }^{1}$, Lori M. Lubin ${ }^{1}$, Alice E. Shapley ${ }^{2}$, Roy R. Gal ${ }^{3}$, and Gordon K. Squires ${ }^{4}$ \\ University of California Observatories/Lick Observatory, University of California, Santa Cruz, CA 95064, USA; kocevski@ucolick.org \\ Received 2010 December 26; accepted 2011 June 13; published 2011 August 4
}

\begin{abstract}
We have employed emission-line diagnostics derived from DEIMOS and NIRSPEC spectroscopy to determine the origin of the $[\mathrm{O}$ II] emission line observed in six active galactic nucleus (AGN) hosts at $z \sim 0.9$. These galaxies are a subsample of AGN hosts detected in the Cl1604 supercluster that exhibit strong Balmer absorption lines in their spectra and appear to be in a post-starburst or post-quenched phase, if not for their [O II] emission. Examining the flux ratio of the [N II] to $\mathrm{H} \alpha$ lines, we find that in five of the six hosts the dominant source of ionizing flux is AGN continuum emission. Furthermore, we find that four of the six galaxies have over twice the [O II] line luminosity that could be generated by star formation alone given their $\mathrm{H} \alpha$ line luminosities. This strongly suggests that AGN-excited narrow-line emission is contaminating the [O II] line flux. A comparison of star formation rates calculated from extinction-corrected $\left[\mathrm{O}_{\mathrm{II}}\right]$ and $\mathrm{H} \alpha$ line luminosities indicates that the former yields a five-fold overestimate of the current activity in these galaxies. Our findings reveal the [O II] line to be a poor indicator of star formation activity in a majority of these moderate-luminosity Seyferts. This result bolsters our previous findings that an increased fraction of AGN at high redshifts is hosted by galaxies in a post-starburst phase. The relatively high fraction of AGN hosts in the Cl1604 supercluster that show signs of recently truncated star formation activity may suggest that AGN feedback plays an increasingly important role in suppressing ongoing activity in large-scale structures at high redshift.
\end{abstract}

Key words: galaxies: active - galaxies: clusters: general - galaxies: evolution - X-rays: galaxies

Online-only material: color figures

\section{INTRODUCTION}

There is growing evidence for a link between the evolution of supermassive black holes (SMBHs) and the galaxies that host them. This includes the observed correlation between SMBH mass and bulge velocity dispersion (Gebhardt et al. 2000; Tremaine et al. 2002). How such correlations are established remains to be determined, but they suggest that SMBHs and their host galaxies play a role in regulating one another's growth. Recent hydrodynamical simulations suggest that feedback from active galactic nuclei (AGNs) may provide a means for this regulation. In this scenario, a sufficiently energetic AGN can drive outflows that disrupt its host galaxy and effectively quench ongoing star formation by removing the galaxy's gas supply (Springel et al. 2005; Hopkins et al. 2005).

If AGNs are responsible for rapidly quenching star formation in their hosts, then signs of recent transformation should be present in these galaxies, including post-starburst spectral signatures. Post-starburst galaxies exhibit Balmer absorption features due to the presence of recently formed $(<1 \mathrm{Gyr})$ A-type stars, but lack emission lines that are indicative of ongoing star formation, such as $\mathrm{H} \alpha$ at $6563 \AA$ or the [O II] doublet at $3727 \AA$. Similar, yet weaker, features are also found in galaxies that have experienced a rapid truncation of normal star formation (i.e., post-quenched galaxies; Poggianti 2004).

Several recent studies have found evidence that post-starburst features are detected in AGN hosts more often than in their non-

\footnotetext{
1 Also at Department of Physics, University of California, Davis, 1 Shields Avenue, Davis, CA 95616, USA.

2 Also at Department of Physics and Astronomy, 430 Portola Plaza, University of California, Los Angeles, CA 90095, USA.

3 Also at Institute for Astronomy, University of Hawaii, 2680 Woodlawn Drive, Honolulu, HI 96822, USA.

4 Also at Spitzer Science Center, M/S 220-6, California Institute of Technology, 1200 East California Boulevard, Pasadena, CA 91125, USA.
}

active counterparts. For example, Goto (2006) finds that $4.2 \%$ of optically identified AGNs at low redshifts exhibit post-starburst spectral signatures, compared to only $0.2 \%$ of normal galaxies. At higher redshifts, Georgakakis et al. (2008) determined that $21 \%$ of X-ray-selected AGN hosts located on the red sequence at $0.68 \leqslant z \leqslant 0.88$ exhibit post-starburst signatures. At slightly higher redshifts, Kocevski et al. (2009b, hereafter Koc09b) report that $\sim 44 \%$ of the X-ray-selected AGNs found in the Cl1604 supercluster at $z \sim 0.9$ show strong Balmer absorption features.

One of the difficulties of extending such work to higher redshifts is that the $\mathrm{H} \alpha$ line moves out of the optical window, forcing surveys to rely on the [OII] doublet as the primary indicator of star formation activity. However, a comprehensive study by Yan et al. (2006) suggests that [O II] emission is a poor indicator of star formation activity in many galaxies. They show that in $91 \%$ of red, early-type galaxies that exhibit [O II] emission, the line originates from AGN activity and not normal star formation processes. This poses significant problems for the correct identification of post-starburst/post-quenched galaxies at high redshift, which are often selected based on the absence of [O II] emission (e.g., Dressler \& Gunn 1983; Poggianti et al. 1999). If AGNs contribute significantly to their host galaxies' [O II] line flux, then excluding all [O II] emitters from such samples would severely underestimate the fraction of AGN hosts that are truly in a post-starburst or post-quenched phase.

In this study, we use emission-line diagnostics measured from optical and near-infrared spectra to investigate the origin of [O II] emission in six X-ray-selected AGNs at $z \sim 0.9$. These AGNs are a subset of active galaxies detected in the Cl1604 supercluster by Koc09b that were found to have both strong Balmer absorption lines indicative of post-starburst/postquenched systems as well as moderate levels of [O II] emission. If the observed [O II] lines in these galaxies are of AGN origin, then the high incidence of Balmer features would indicate an 
Table 1

Properties of the C11604 AGN Sample Observed with NIRSPEC

\begin{tabular}{|c|c|c|c|c|c|c|c|c|}
\hline ID & Name & $\begin{array}{l}\text { R.A. } \\
\text { (J2000) }\end{array}$ & $\begin{array}{c}\text { Decl. } \\
(\mathrm{J} 2000)\end{array}$ & $z$ & $\begin{array}{c}L_{\mathrm{x}}(0.5-8 \mathrm{keV}) \\
\left(\times 10^{43}\right)^{\mathrm{a}}\end{array}$ & {$[\mathrm{O}$ II $] \mathrm{EW}$} & $\mathrm{H} \alpha \mathrm{EW}$ & $\log (F[\mathrm{~N} \mathrm{II}] / F[\mathrm{H} \alpha])$ \\
\hline X0 & $\mathrm{J} 160415.6+431016$ & 241.06492 & 43.17134 & 0.900 & 4.47 & -17.8 & -33.3 & -0.49 \\
\hline $\mathrm{X} 1$ & $\mathrm{~J} 160425.9+431245$ & 241.10783 & 43.21265 & 0.871 & 1.31 & -31.3 & -2.5 & $>-0.12^{b}$ \\
\hline $\mathrm{X} 2$ & $\mathrm{~J} 160423.9+431125$ & 241.09959 & 43.19052 & 0.867 & 3.39 & -7.3 & -16.5 & -0.22 \\
\hline X3 & $\mathrm{J} 160436.7+432141$ & 241.15290 & 43.36147 & 0.923 & 2.28 & -7.1 & -20.8 & -0.06 \\
\hline $\mathrm{X} 4$ & $\mathrm{~J} 160408.2+431736$ & 241.03416 & 43.29359 & 0.937 & 2.75 & -8.0 & -5.7 & 0.60 \\
\hline X5 & $\mathrm{J} 160401.3+431351$ & 241.00546 & 43.23089 & 0.927 & 3.44 & -3.3 & -2.8 & $>0.55^{\mathrm{b}}$ \\
\hline
\end{tabular}

Notes.

${ }^{a}$ In units of $h_{70}^{-2} \mathrm{erg} \mathrm{s}^{-1}$.

b $3 \sigma$ lower limit.

increased post-starburst fraction among AGN hosts at high redshift. Using newly obtained Keck II Near-Infrared Echelle Spectrograph (NIRSPEC) spectroscopy, we examine the flux ratio of the $[\mathrm{N}$ II] $\lambda 6584$ to $\mathrm{H} \alpha$ lines to ascertain the source of the ionizing flux exciting the [O II] emission found in these galaxies.

We present our analysis in the following manner. Section 2 describes the C11604 supercluster and our AGN sample in greater detail and Section 3 discusses the observation and reduction of the near-infrared spectroscopy. In Section 4, we describe our methods for measuring equivalent widths (EWs) and line flux ratios. In Section 5 we discuss our findings, while in Section 6 we summarize our conclusions.

\section{THE Cl1604 AGN SAMPLE}

The six AGNs targeted for NIRSPEC observations are spectroscopically confirmed members of the $\mathrm{Cl} 1604$ supercluster, a large-scale structure which consists of eight galaxy clusters and groups at a median redshift of $z \sim 0.9$ (Gal \& Lubin 2004). The supercluster has been extensively studied at a variety of wavelengths (Gal et al. 2008; Kocevski et al. 2009a, 2011; L. M. Lubin et al. 2011, in preparation) and in Koc09b we identified nine X-ray-selected AGNs within the $\mathrm{Cl1604}$ system. These AGNs have moderate X-ray luminosities $\left(L_{\mathrm{X}} \sim 10^{43} \mathrm{erg} \mathrm{s}^{-1}\right)$ and their optical spectra are consistent with type 2 Seyferts. The average spectral properties of the C11604 AGNs targeted with NIRSPEC can be seen in Figure 1, where a high signalto-noise ratio $(\mathrm{S} / \mathrm{N})$ composite spectrum created by co-adding the individual AGN spectra is shown. All of the AGNs exhibit moderate to strong [O II] emission lines and a majority show strong Balmer absorption features, which are clearly visible in the stacked spectra.

Our available observing time allowed for only two-thirds of the full Cl1604 X-ray AGN sample to be targeted with NIRSPEC. Preference was given to those galaxies whose $\mathrm{H} \alpha$ and $[\mathrm{NII}]$ features would be detected blueward of the strong $\mathrm{OH}$ airglow lines at $\lambda \approx 1.27 \mu \mathrm{m}(z \leqslant 0.93)$. The details of the NIRSPEC observations are given in Section 3.2. The coordinates, redshifts, X-ray luminosities, and spectral properties of the six C11604 AGNs observed with NIRSPEC are listed in Table 1.

\section{OBSERVATIONS AND DATA REDUCTION}

\subsection{Optical Spectroscopy}

The vast majority of redshifts in the $\mathrm{Cl1604}$ field come from observations of 18 slit masks with DEIMOS on the Keck II telescope between 2003 May and 2010 June (Gal et al. 2008).
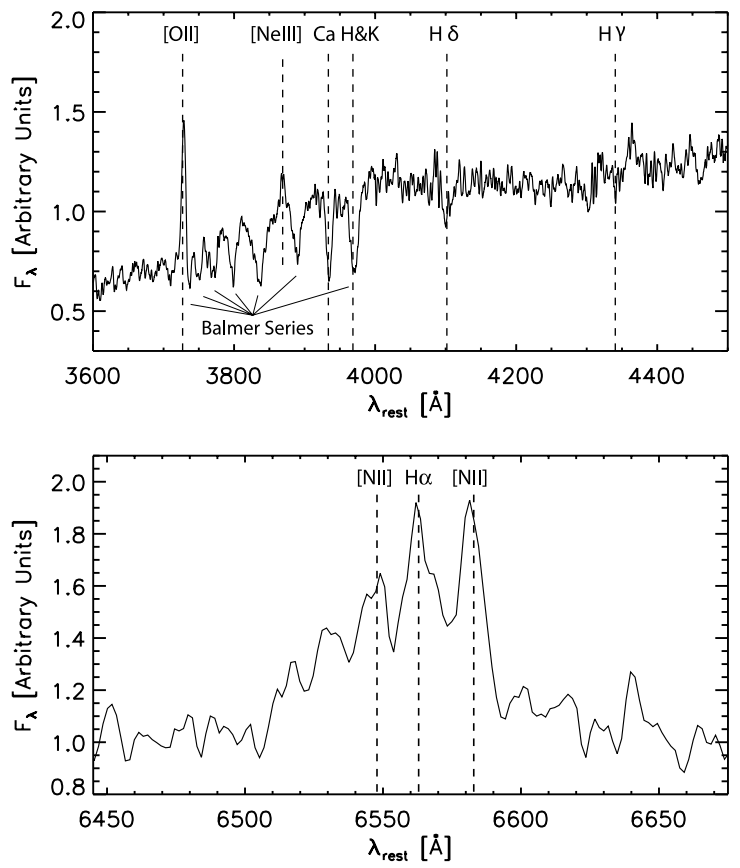

Figure 1. Stacked DEIMOS (above) and NIRSPEC (below) rest-frame composite spectra of the Cl1604 AGN sample.

Slit masks were observed with the $1200 \mathrm{l} \mathrm{mm}^{-1}$ grating with an FWHM resolution of $\sim 1.7 \AA\left(68 \mathrm{~km} \mathrm{~s}^{-1}\right)$ and a typical wavelength coverage of $6385-9015 \AA$. The exposure frames for each slit mask were reduced using the DEEP2 version of the spec $2 d$ package (Davis et al. 2003) as described in Lemaux et al. (2009).

In total, 1339 high-quality extragalactic DEIMOS spectra were obtained in the $\mathrm{Cl1604}$ field, with 432 objects having measured redshifts within the adopted redshift range of the supercluster. Combined with additional redshifts obtained with the Low-Resolution Imaging Spectrometer (Oke et al. 1995), 517 high-quality spectra have been obtained for members of the C11604 supercluster.

\subsection{Near-infrared Spectroscopy}

To measure the $\mathrm{H} \alpha$ and [N II] emission lines we used the NIRSPEC spectrograph (McLean et al. 1998) on the Keck II telescope. Five of our Cl1604 X-ray AGNs were targeted in 2009 June and one in 2007 June as part of the sample in Lemaux et al. (2010, hereafter Lem10). Observations were taken in lowresolution mode with slit widths of 0.76 , resulting in a pixel scale of $3 \AA$ pixel $^{-1}$ and an FWHM resolution of $\sim 8 \AA$. The observations were taken through the NIRSPEC-3 filter, with a 
typical wavelength coverage of $2900 \AA$ and central wavelength of $1.273 \mu \mathrm{m}$. Conditions were photometric and seeing ranged from $0.55-0.7$.

The observation of each setup consisted of staggered exposures of 300-900 s between nods on the sky of 1".4-2.'5 along the $42^{\prime \prime}$ slit, with total integration times varying between 1800 and $3600 \mathrm{~s}$. Two standard stars drawn from the UKIRT list of bright standards ${ }^{5}$ were observed: HD105601 (A2) at evening twilight and HD203856 (A0) at morning twilight.

The NIRSPEC data were reduced using a publicly available semi-automated Interactive Data Language pipeline (G. Becker 2010, private communication). This pipeline is used to generate flat-fielded, dark-subtracted, cosmic-ray-cleaned, wavelengthand flux-calibrated one- and two-dimensional spectra. Further details of the reduction process are given in Lem10. Composite NIRSPEC and DEIMOS spectra of the six C11604 AGN hosts, created by averaging the spectra of the individual galaxies, are shown in the two panels of Figure 1.

\section{ANALYSIS}

\subsection{Spectral Line Measurements}

In each processed one-dimensional DEIMOS and NIRSPEC spectrum, we measure the rest-frame EW of the [O II] and $\mathrm{H} \alpha$ nebular emission features using two techniques: bandpass measurements and line-fitting techniques. Bandpass measurements were performed on all spectra by integrating around the vicinity of a spectral feature using identical bandpass endpoints to those in Lem10. Line fitting was performed on all spectra where emission lines were detected at a significance of greater than $3 \sigma$. For each spectrum, the EW measurement was chosen from the better of the two methods, typically depending on the feature $S / N$. Emission line fluxes of the [O II], $\mathrm{H} \alpha$, and [N II] features were calculated using the same two methods as those used for EW measurements. While we include EW measurements detected in emission at a significance less than $3 \sigma$ in our analyses, line fluxes measured at low significance were assigned $3 \sigma$ upper limits. Errors are calculated either through a combination of the covariance matrix and Poisson errors (for bandpass measurements) or simply through the covariance matrix of the fit (for line-fitting techniques).

Absolute flux calibration and slit throughput corrections for the Cl1604 DEIMOS and NIRSPEC data were obtained in a manner nearly identical to that of Lemaux et al. (2009) and Lem10, respectively. The NIRSPEC calibration involved comparing observations of the standard star HD203856 with a scaled flux calibrated spectrum of $\alpha$ Lyr (Colina et al. 1996). Following the analysis of Lem10, an internal extinction of $E(B-V)=0.3$ was adopted for all galaxies in our sample and corrected for using the Calzetti et al. (2000) reddening law.

\subsection{Line Ratio Diagnostics}

Several sources of photoionizing radiation can give rise to narrow emission lines in galaxies, including young stellar populations and AGN-related continuum emission. Fortunately, the different line species these two mechanisms excite provide a means to distinguish which is the dominant source of ionizing flux. Therefore, it has become common to use the relative strength of lines with high and low ionization potentials to differentiate these two excitation mechanisms.

\footnotetext{
5 http://www.jach.hawaii.edu/UKIRT/astronomy/calib/phot_cal/ ukirt_stds.html
}

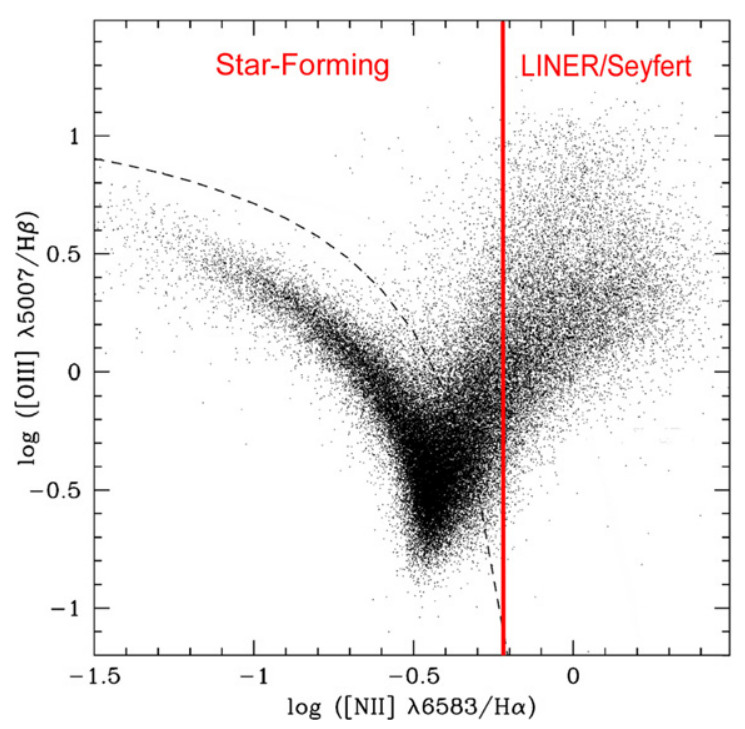

Figure 2. Figure adopted from Kauffmann et al. (2003) showing the emission line flux ratios for 55,000 SDSS galaxies. The dashed line denotes the dividing line between star-forming galaxies and those with emission from a LINER or a Seyfert defined by Kauffmann et al. The red solid vertical line shows our adopted boundary of $\log (F[\mathrm{NII}] / F[\mathrm{H} \alpha])=-0.22$ between star-forming and LINER/Seyfert galaxies.

(A color version of this figure is available in the online journal.)

Introduced by Baldwin et al. (1981, hereafter BPT), such line ratio diagnostics typically utilize the strength of the [O III] feature relative to $\mathrm{H} \beta$ and the strength of a forbidden line (typically $6300 \AA$ [O I], [N II], or $6716 \AA+6731 \AA$ [S II]) relative to $\mathrm{H} \alpha$. An example of a BPT diagram using the $[\mathrm{N} \mathrm{II}] / \mathrm{H} \alpha$ and $[\mathrm{O} \mathrm{III}] / \mathrm{H} \beta$ line flux ratios as measured in $\sim 55,000$ Sloan Digital Sky Survey (SDSS) galaxies is shown in Figure 2. In this parameter space, Seyferts and low-ionization nuclear emissionline regions (LINERs) dominated sources (hereafter LINER/ Seyfert) have higher values of $[\mathrm{N} \mathrm{II}] / \mathrm{H} \alpha$. In a study of nearly 100,000 SDSS galaxies, Kewley et al. (2006) found that the logarithm of the $F([\mathrm{~N} \mathrm{II}]) / F(\mathrm{H} \alpha)$ ratio (hereafter $F_{[\mathrm{NII} / \mathrm{H} \alpha}$ ) for star-forming galaxies varied from -1.5 in extremely metalpoor systems to -0.3 in those with super-solar abundances. This upper bound is similar to the maximal boundary of $\left.\log \left(F_{[\mathrm{N}} \mathrm{II}\right] / \mathrm{H} \alpha\right)=-0.22$ found for star-forming galaxies in a sample of Kauffmann et al. (2003); this limit is denoted by the vertical line in Figure 2.

For this study, we lack observations of the $\mathrm{H} \beta$ and [O III] emission features due to the redshift of our target galaxies. Instead, we rely on a pseudo-BPT diagram that utilizes the $F_{[\mathrm{N} \text { II } / \mathrm{H} \alpha}$ ratio as the primary discriminator between star-forming galaxies and galaxies dominated by LINER/Seyfert emission. In addition, we use the EW of the [O II] and $\mathrm{H} \alpha$ emission features as a replacement for the traditional ordinate of the BPT diagram (i.e., $F([\mathrm{O} \mathrm{III}]) / F(\mathrm{H} \beta)$ ). Yan et al. (2006) previously demonstrated the effectiveness of this type of pseudo-BPT diagram in separating star-forming and AGN-like systems (they utilized the $[\mathrm{N}$ II $] / \mathrm{H} \alpha$ and $[\mathrm{O} \mathrm{II}] / \mathrm{H} \beta$ ratios; see their Figure 11).

Without the additional information that $F_{[\mathrm{OII}] / \mathrm{H} \beta}$ provides, we have chosen to divide star-forming galaxies from those dominated by a LINER/Seyfert component at the Kauffmann et al. (2003) $\operatorname{limit}$ of $\log \left(F_{[\mathrm{NII} / \mathrm{H} \alpha}\right)=-0.22$. This value is similar to cuts used in other studies when $\mathrm{H} \beta$ and [O III] are weak or unobservable (e.g., Miller et al. 2003; Stasińska et al. 2006; Lem10). Furthermore, we believe this value to be a conservative boundary between star-forming galaxies and LINER/Seyferts 


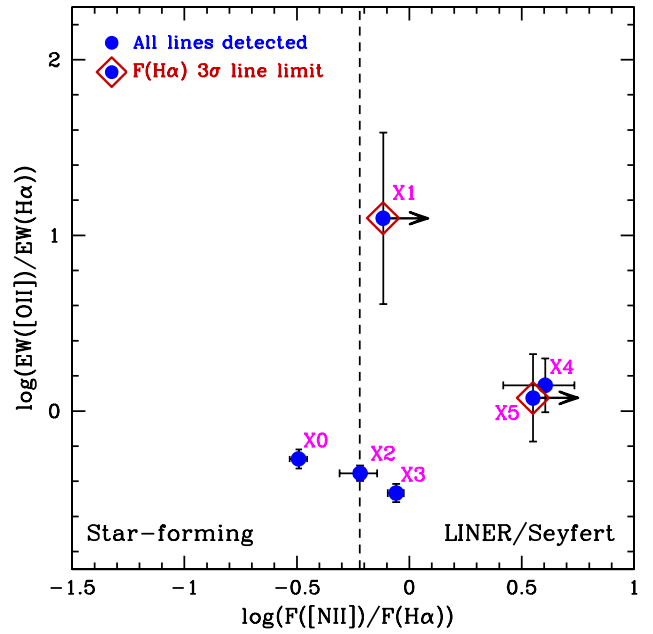

Figure 3. Ratio of $[\mathrm{O}$ II] and $\mathrm{H} \alpha$ EWs as a function of $F([\mathrm{~N}$ II] $]) / F(\mathrm{H} \alpha)$ for the six Cl1604 AGNs observed with NIRSPEC. Galaxies with $3 \sigma$ upper limits on $F(\mathrm{H} \alpha)$ are plotted with arrows. The vertical dashed line at $\log (F[\mathrm{NII}] / F[\mathrm{H} \alpha]=$ -0.22 indicates our dividing line between star-forming and LINER/Seyfert galaxies.

(A color version of this figure is available in the online journal.)

as previous studies have found evidence for substantial AGN contamination out to as far as $\log \left(F_{[\mathrm{N} \mathrm{II]} / \mathrm{H} \alpha}\right)=-0.35$ (Stasińska et al. 2006). In the following section we discuss where the six Cl1604 X-ray AGNs targeted with NIRSPEC fall on our pseudoBPT diagram.

\section{RESULTS}

In Figure 3, we plot the ratio of [O II] and $\mathrm{H} \alpha$ rest-frame EWs as a function of $F_{[\mathrm{NII}] / \mathrm{H} \alpha}$ for the six Cl1604 AGN hosts observed with NIRSPEC. In four of these six galaxies we detect strong $(>3 \sigma)$ [O II], [N II], and $\mathrm{H} \alpha$ emission lines, while in two galaxies (X1 and X5) [N II] was detected in our NIRSPEC spectroscopy, but $\mathrm{H} \alpha$ was not. For these two galaxies we make use of $3 \sigma$ upper limits on the flux and luminosity of the $\mathrm{H} \alpha$ line. We find that five of the six $\mathrm{Cl} 1604$ AGN hosts have $[\mathrm{N} \mathrm{II}] / \mathrm{H} \alpha$ flux ratios greater than $\log \left(F_{[\mathrm{N} \mathrm{III} / \mathrm{H} \alpha}\right)=-0.22$, the maximum value found for star-forming galaxies by Kauffmann et al. (2003). This suggests that the dominant source of ionizing flux in these galaxies is AGN-related emission and not radiation from young stellar populations.

The single Cl1604 AGN found to lie in the star-forming region of our pseudo-BPT diagram is hosted by a spiral galaxy with clear signs of ongoing star formation within its disk. This galaxy has the latest morphological type among the $\mathrm{Cl} 1604$ AGN hosts. An ACS-F814W image of the galaxy can be seen in Figure 4 of Koc $09 \mathrm{~b}$ (see source 5). The galaxy also appears to be in the early stages of an ongoing merger, as it has a nearby companion that is spectroscopically confirmed to be at the same redshift (Koc09b). Given these properties, it is not surprising that our emission-line diagnostic indicates the presence of ongoing star formation in the system.

To further investigate whether the [O $\mathrm{II}]$ emission lines observed in these galaxies could originate from star formation processes, we compared the extinction-corrected [O II] and $\mathrm{H} \alpha$ line luminosities measured in each galaxy. The $L([\mathrm{O} \mathrm{II}]) / L(\mathrm{H} \alpha)$ (hereafter $L_{[\mathrm{OII}] / \mathrm{H} \alpha}$ ) ratio, plotted against $F_{[\mathrm{NII}] / \mathrm{H} \alpha}$, is shown in Figure 4. Kewley et al. (2004) previously demonstrated that the maximum $L_{[\mathrm{OII} / \mathrm{H} \alpha}$ ratio that can be achieved by star-forming regions is roughly 2.1. We find that four of the six Cl1604 AGN hosts have $L_{[\mathrm{O} \text { II] } / \mathrm{H} \alpha}$ ratios that exceed this limit. On average, these galaxies have over twice the [O II] line luminosity that could be generated by star formation processes alone given their observed $\mathrm{H} \alpha$ line luminosity. The two galaxies that fall below the Kewley et al. maximum are sources X0, the star-forming spiral host previously discussed, and source X3. This latter source is the only Cl1604 AGN in which the highly ionized [Ne v] emission line is visible. The strength of the [O II] line is predicted and observed to decrease in high-ionization AGNs (Ho 2005 ), which likely explains the lack of [O II] contamination in this galaxy.

To determine the degree to which current star formation activity would be overestimated in the galaxies showing excess

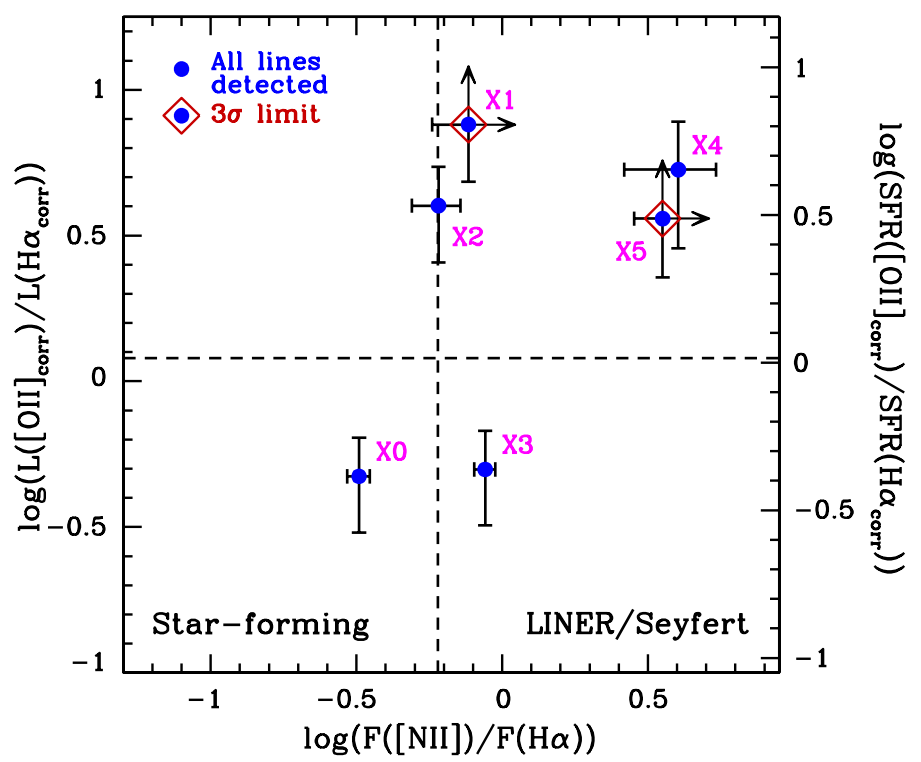

Figure 4. Ratio of extinction-corrected $L\left(\left[\mathrm{O}_{\mathrm{II}}\right]\right)$ and $L(\mathrm{H} \alpha)$ as a function of $\log \left(F\left[\mathrm{~N}_{\mathrm{II}}\right] / F[\mathrm{H} \alpha]\right)$ for the Cl1604 AGN sample. Galaxies with $3 \sigma$ upper limits on $F(\mathrm{H} \alpha)$ and $L(\mathrm{H} \alpha)$ are plotted with arrows. The vertical dashed line at $F[\mathrm{~N} I I] / F[\mathrm{H} \alpha]=-0.22$ denotes our boundary between a star-forming and a LINER/Seyfert classification. The horizontal dashed line at $\log (L[\mathrm{O} \mathrm{II}] / L[\mathrm{H} \alpha])=0.08$ is the average extinction-corrected luminosity ratio for star-forming galaxies at low redshift (Kewley et al. 2004).

(A color version of this figure is available in the online journal.) 
[O II] emission, we calculated the star formation rate (SFR) of each host using the relations of Kennicutt (1998) and the observed [O II] and $\mathrm{H} \alpha$ line luminosities. On the right axis of Figure 4, we compare the SFR derived from the extinctioncorrected $\mathrm{H} \alpha$ line to that of the extinction-corrected [O II] line using a constant $E(B-V)=0.3$ (adopted from Lemaux et al. 2010). In the four AGN hosts where [O II] is elevated relative to $\mathrm{H} \alpha$, the SFR would, on average, be overestimated by a factor of five. We find that varying the assumed extinction over a reasonable range of $E(B-V)$ values (i.e., $0.1-0.55)$ does not significantly change this result. Even if we make no correction for extinction, which attenuates [O II] more than $\mathrm{H} \alpha$, the [O II]derived SFRs in these galaxies would be over twice the rate derived from $\mathrm{H} \alpha$.

Our finding that the [O II] line can be significantly contaminated bolster the results of Koc09b, who reported that a large fraction of AGNs in the Cl1604 supercluster appear to be associated with recently quenched hosts. A major caveat in that study was the assumption that the observed [O II] emission in the spectra of these galaxies was due to AGN activity and not ongoing star formation. We have now demonstrated that this assumption is supported by our new observations. This finding provides significant insight into the nature of these systems. In the absence of substantial star formation, the properties of these galaxies generally agree with AGN feedback scenarios in which AGN-driven outflows rapidly suppress the star formation activity of their hosts. For example, we find both a high incidence of Balmer lines and a large $\mathrm{A} / \mathrm{K}$ ratio $^{6}$ in the composite spectra of the $\mathrm{Cl1604}$ hosts, which suggests that these systems are either in a post-starburst phase or have recently had their star formation rapidly truncated. Furthermore, these galaxies have optical colors that place them between the red sequence of earlytype galaxies and the blue cloud of star-forming objects, while their morphologies show tell-tale signs of interactions in the recent past, including fading tidal features and multiple nuclei. In most regards, these galaxies resemble a transition population within the $\mathrm{Cl} 1604$ structure that has been caught in the process of transforming from actively star-forming galaxies to passively evolving systems. The fact that a large fraction of AGNs within the Cl1604 supercluster show such signatures, coupled with recent findings that high-redshift AGNs are more common in denser environments than they are in the field (Gilli et al. 2003; Cappelluti et al. 2005; Eastman et al. 2007; Kocevski et al. 2009a), suggests that AGN-related feedback may play an important role in accelerating galaxy evolution in large-scale structures at high redshift.

\section{CONCLUSIONS}

We have used emission-line ratios measured from DEIMOS and NIRSPEC spectroscopy to determine the origin of the [O II] emission line observed in six AGN hosts at $z \sim 0.9$. These six galaxies are a subsample of nine AGNs detected in the $\mathrm{Cl1604}$ supercluster that exhibit strong Balmer absorption lines in their spectra. If not for the presence of the [O II] line, many of these galaxies would be classified as post-starburst or post-quenched systems.

Examining the flux ratio of the $\left[\mathrm{N}_{\mathrm{II}}\right]$ to $\mathrm{H} \alpha$ lines, we find that in five of the six AGN hosts the dominant source of ionizing flux is AGN continuum emission and not radiation from young

\footnotetext{
6 The A/K ratio measures the ratio of the young and old stellar components in a spectrum as measured largely by the Balmer absorption line strengths and the strength of the $4000 \AA$ break (e.g., Yan et al. 2006).
}

stellar populations. Furthermore, we find that four of the six galaxies have, on average, over twice the [O II] line luminosity that could be generated by star formation processes alone given their $\mathrm{H} \alpha$ line luminosities. This strongly suggests that AGNexcited narrow-line emission is contaminating the [O II] line flux. A comparison of SFRs calculated from the [O II] and $\mathrm{H} \alpha$ line luminosities indicates that the former yields a five-fold overestimate of the star formation activity of these galaxies.

Our findings reveal the [O II] line to be a poor indicator of current star formation activity in a majority of these moderateluminosity Seyferts; a result that agrees with the conclusions of Yan et al. (2006) and Lem10. This has important ramifications on the study of post-starburst and recently quenched AGN hosts at high redshifts. Since post-starburst systems are selected based on the absence of current star formation, any studies that rely solely on the [O II] line as a measure of current activity in AGN hosts will severely underestimate the fraction of AGNs found in post-starburst galaxies. Furthermore, our results confirm the increased connection between AGN activity and post-starburst/ post-quenched systems at high redshifts reported by Koc09b. Although we do not observe direct evidence for AGN-driven quenching, the relatively high fraction of AGN hosts in the Cl1604 supercluster that shows signs of recently truncated star formation activity may suggest that AGN feedback plays an increasingly important role in suppressing ongoing activity and accelerating galaxy evolution in overdense environments at high redshift.

\section{REFERENCES}

Baldwin, J. A., Phillips, M. M., \& Terlevich, R. 1981, PASP, 93, 5 Calzetti, D., Armus, L., Bohlin, R. C., et al. 2000, ApJ, 533, 682 Cappelluti, N., Cappi, M., Dadina, M., et al. 2005, A\&A, 430, 39 Colina, L., Bohlin, R. C., \& Castelli, F. 1996, AJ, 112, 307

Davis, M., Faber, S. M., Newman, J., et al. 2003, Proc. SPIE, 4834, 161 Dressler, A., \& Gunn, J. E. 1983, ApJ, 270, 7

Eastman, J., Martini, P., Sivakoff, G., et al. 2007, ApJ, 664, L9

Gal, R. R., Lemaux, B. C., Lubin, L. M., Kocevksi, D., \& Squires, G. K. 2008, ApJ, 684, 933

Gal, R. R., \& Lubin, L. M. 2004, ApJ, 607, L1

Gebhardt, K., Bender, R., Bower, G., et al. 2000, ApJ, 539, L13

Georgakakis, A., Nandra, K., Yan, R., et al. 2008, MNRAS, 385, 2049

Gilli, R., Cimatti, A., Daddi, E., et al. 2003, ApJ, 592, 721

Goto, T. 2006, MNRAS, 369, 1765

Ho, L. C. 2005, ApJ, 629, 680

Hopkins, P. F., Hernquist, L., Martini, P., et al. 2005, ApJ, 625, L71

Kauffmann, G., Heckman, T. M., Tremonti, C., et al. 2003, MNRAS, 346, 1055 Kennicutt, R. C., Jr. 1998, ARA\&A, 36, 189

Kewley, L. J., Geller, M. J., \& Jansen, R. A. 2004, AJ, 127, 2002

Kewley, L. J., Groves, B., Kauffmann, G., \& Heckman, T. 2006, MNRAS, 372 , 961

Kocevski, D. D., Lemaux, B. C., Lubin, L. M., et al. 2011, ApJ, 738, 38

Kocevski, D. D., Lubin, L. M., Gal, R., et al. 2009a, ApJ, 690, 295

Kocevski, D. D., Lubin, L. M., Lemaux, B. C., et al. 2009b, ApJ, 700, 901

Lemaux, B. C., Lubin, L. M., Sawicki, M., et al. 2009, ApJ, 700, 20

Lemaux, B. C., Lubin, L. M., Shapley, A., et al. 2010, ApJ, 716, 970

McLean, I. S., Becklin, E. E., Bendiksen, O., et al. 1998, Proc. SPIE, 3354, 566

Miller, C. J., Nichol, R. C., Gómez, P. L., Hopkins, A. M., \& Bernardi, M. 2003, ApJ, 597, 142

Oke, J. B., Cohen, J. G., Carr, M., et al. 1995, PASP, 107, 375

Poggianti, B. M. 2004, in Clusters of Galaxies: Probes of Cosmological Structure and Galaxy Evolution, ed. J. S. Mulchaey, A. Dressler, \& A. Oemler (Carnegie Obs. Astrophys. Ser. Vol. 3; Cambridge: Cambridge Univ Press), 245

Poggianti, B. M., Bridges, T. J., Komiyama, Y., et al. 2004, ApJ, 601, 197

Poggianti, B. M., Smail, I., Dressler, A., et al. 1999, ApJ, 518, 576

Springel, V., Di Matteo, T., \& Hernquist, L. 2005, ApJ, 620, L79

Stasińska, G., Cid Fernandes, R., Mateus, A., Sodré, L., \& Asari, N. V. 2006, MNRAS, 371, 972

Tremaine, S., Gebhardt, K., Bender, R., et al. 2002, ApJ, 574, 740

Yan, R., Newman, J. A., Faber, S. M., et al. 2006, ApJ, 648, 281 